\title{
TIME-OPTIMAL CONTROL OF INFINITE ORDER HYPERBOLIC SYSTEMS WITH TIME DELAYS
}

\author{
ADAM KOWALEWSKI \\ Institute of Automatics \\ AGH University of Science and Technology, Al. Mickiewicza 30, 30-059 Cracow, Poland \\ e-mail:ako@ia.agh.edu.pl
}

\begin{abstract}
In this paper, the time-optimal control problem for infinite order hyperbolic systems in which time delays appear in the integral form both in state equations and in boundary conditions is considered. Optimal controls are characterized in terms of an adjoint system and shown to be unique and bang-bang. These results extend to certain cases of nonlinear control problems. The particular properties of optimal control are discussed.
\end{abstract}

Keywords: time-optimal control, infinite order, hyperbolic systems, time delays.

\section{Introduction}

Various optimization problems associated with optimal control of second order distributed parameter systems with time delays appearing in boundary conditions were studied in (Wang, 1975; Knowles, 1978; Kowalewski, 1993a; 1993b; 1995; 1998; 2000; 2003).

In (Knowles, 1978), time optimal control problems of linear parabolic systems with Neumann boundary conditions involving constant time delays were considered.

These equations constitute, in a linear approximation, a universal mathematical model for many diffusion processes in which time-delayed feedback signals are introduced at the boundary of a system's spatial domain. For example, in the area of plasma control (Wang, 1975), it is of interest to confine a plasma in a given bounded spatial domain $\Omega$ by introducing a finite electric potential barrier or a "magnetic mirror" surrounding $\Omega$. For a collisiondominated plasma, its particle density is describable by a parabolic equation. Due to particle inertia and the finiteness of the electrical potential barrier or the magneticmirror field strength, the particle reflection at the domain boundary is not instantaneous. Consequently, the particle flux at the boundary of $\Omega$ at any time depends on the flux of particles which escaped earlier and reflected back into $\Omega$ at a later time. This leads to boundary conditions involving time delays.

Using the results of (Wang, 1975), the existence of a unique solution of such parabolic systems was discussed. A characterization of optimal control in terms of the ad- joint system was given. Consequently, this characterization was used to derive specific properties of optimal control (bang-bangness, uniqueness, etc.). These results were also extended to certain cases of nonlinear control without convexity and to certain fixed-time problems.

Consequently, in (Kowalewski, 1993a; 1993b; 1995; 1998; 2000), linear quadratic problems for hyperbolic systems with time delays given in various forms (constant time delays, time-varying delays, integral time delays, etc.) were presented.

In particular, in (Kowalewski, 2003), time-optimal control problems for second order hyperbolic systems with deviating arguments appearing in the integral form both in state equations and in Neumann boundary conditions were considered. The presented minimum time problem can be generalized onto the case of time-delay infinite order hyperbolic systems. For this reason, in the present paper we consider the time-optimal control problem for linear infinite order hyperbolic systems in which time delays appear in the integral form both in state equations and in Neumann boundary conditions.

Such hyperbolic systems constitute in a linear approximation mathematical models of representative convection-reaction processes, e.g., fixed-bed reactors, pressure swing absorbtion processes, etc.

We consider a different type of equations, namely, infinite order partial differential equations of hyperbolic type and a new type of time delays given in the integral form. 
The existence and uniqueness of solutions for such hyperbolic equations were proved-Lemma 1 and Theorem 11. Optimal control is characterized by the adjoint problem-Lemma2 and Theorem 2. By using this characterization, particular properties of optimal control are proved, i.e., uniqueness and the bang-bang propertyTheorem 3 and 4

Moreover, the time-optimal control problems presented in this paper are extended to certain cases of nonlinear control without the assumption about convexityTheorem 5. Examples of application are also presented.

\section{Preliminaries}

Let $\Omega$ be a bounded open set of $\mathbb{R}^{n}$ with smooth boundary $\Gamma$. We define the infinite order Sobolev space $H^{\infty}\left\{a_{\alpha}, 2\right\}(\Omega)$ of functions $\Phi(x)$ defined on $\Omega$ (Dubinskij, 1975; 1976) as follows:

$$
\begin{aligned}
& H^{\infty}\left\{a_{\alpha}, 2\right\}(\Omega) \\
& =\left\{\Phi(x) \in C^{\infty}(\Omega): \sum_{|\alpha|=0}^{\infty} a_{\alpha}\left\|\mathcal{D}^{\alpha} \Phi\right\|_{2}^{2}<\infty\right\},
\end{aligned}
$$

where $C^{\infty}(\Omega)$ is the space of infinitely differentiable functions, $a_{\alpha} \geq 0$ is a numerical sequence and $\|\cdot\|_{2}$ is the norm in the space $L^{2}(\Omega)$, and

$$
\mathcal{D}^{\alpha}=\frac{\partial^{|\alpha|}}{\left(\partial x_{1}\right)^{\alpha_{1}} \ldots\left(\partial x_{n}\right)^{\alpha_{n}}},
$$

$\alpha=\left(\alpha_{1}, \ldots, \alpha_{n}\right)$ being a multi-index for differentiation, $|\alpha|=\sum_{i=1}^{n} \alpha_{i}$.

The space $H^{-\infty}\left\{a_{\alpha}, 2\right\}(\Omega)$ (Dubinskij, 1975; 1976) is defined as the formal conjugate space to the space $H^{\infty}\left\{a_{\alpha}, 2\right\}(\Omega)$, namely,

$$
\begin{aligned}
& H^{-\infty}\left\{a_{\alpha}, 2\right\}(\Omega) \\
& =\left\{\Psi(x): \Psi(x)=\sum_{|\alpha|=0}^{\infty}(-1)^{|\alpha|} a_{\alpha} \mathcal{D}^{\alpha} \Psi_{\alpha}(x)\right\},
\end{aligned}
$$

where $\Psi_{\alpha} \in L^{2}(\Omega)$ and

$$
\sum_{|\alpha|=0}^{\infty} a_{\alpha}\left\|\Psi_{\alpha}\right\|_{2}^{2}<\infty .
$$

The duality pairing of the spaces $H^{\infty}\left\{a_{\alpha}, 2\right\}(\Omega)$ and $H^{-\infty}\left\{a_{\alpha}, 2\right\}(\Omega)$ is postulated by the formula

$$
\langle\Phi, \Psi\rangle=\sum_{|\alpha|=0}^{\infty} a_{\alpha} \int_{\Omega} \Psi_{\alpha}(x) \mathcal{D}^{\alpha} \Phi(x) \mathrm{d} x
$$

where $\Phi \in H^{\infty}\left\{a_{\alpha}, 2\right\}(\Omega), \Psi \in H^{-\infty}\left\{a_{\alpha}, 2\right\}(\Omega)$.
From the above, $H^{\infty}\left\{a_{\alpha}, 2\right\}(\Omega)$ is everywhere dense in $L^{2}(\Omega)$ with topological inclusions and $H^{-\infty}\left\{a_{\alpha}, 2\right\}(\Omega)$ denotes the topological dual space with respect to $L^{2}(\Omega)$, so we have the following chain:

$$
H^{\infty}\left\{a_{\alpha}, 2\right\}(\Omega) \subseteq L^{2}(\Omega) \subseteq H^{-\infty}\left\{a_{\alpha}, 2\right\}(\Omega) .
$$

\section{Existence and uniqueness of solutions}

Consider now the distributed-parameter system described by the following hyperbolic delay equation:

$$
\begin{gathered}
\frac{\partial^{2} y}{\partial t^{2}}+A y+\int_{a}^{b} c(x, t) y(x, t-h) \mathrm{d} h=u, \\
x \in \Omega, t \in(0, T), \\
y\left(x, t^{\prime}\right)=\Phi_{0}\left(x, t^{\prime}\right), \quad x \in \Omega, t^{\prime} \in[-b, 0), \\
y(x, 0)=y_{p}(x), \quad x \in \Omega, \\
y^{\prime}(x, 0)=y_{I}(x), \quad x \in \Omega, \\
\frac{\partial y}{\partial \eta_{A}}=\int_{a}^{b} d(x, t) y(x, t-h) \mathrm{d} h+v, \\
y\left(x, t^{\prime}\right)=\Psi_{0}\left(x, t^{\prime}\right), \quad x \in \Gamma, t \in(0, T),
\end{gathered}
$$

where $\Omega$ has the same properties as in Section 2,

$$
\begin{gathered}
y \equiv y(x, t ; u), \quad u \equiv u(x, t), \quad v \equiv v(x, t), \\
Q \equiv \Omega \times(0, T), \quad \bar{Q}=\bar{\Omega} \times[0, T], \quad Q_{0}=\Omega \times[-b, 0), \\
\Sigma=\Gamma \times(0, T), \quad \Sigma_{0}=\Gamma \times[-b, 0),
\end{gathered}
$$

- $y$ is a function defined on $Q$ such that $\Omega \times(0, T) \ni$ $(x, t) \mapsto y(x, t) \in \mathbb{R}$,

- $u, v$ are functions defined on $Q$ and $\Sigma$ such that $\Omega \times(0, T) \ni(x, t) \mapsto u(x, t) \in \mathbb{R}$ and $\Gamma \times(0, T) \ni(x, t) \mapsto v(x, t) \in \mathbb{R}$, respectively,

- $c$ is a given real $C^{\infty}$ function defined on $\bar{Q}$,

- $d$ is a given real $C^{\infty}$ function defined on $\Sigma$,

- $h$ is a time delay such that $h \in(a, b)$ and $a>0$,

- $\Phi_{0}, \Psi_{0}$ are initial functions defined on $Q_{0}$ and $\Sigma_{0}$ such that

$\Omega \times[-b, 0) \ni\left(x, t^{\prime}\right) \mapsto \Phi_{0}\left(x, t^{\prime}\right) \in \mathbb{R}$, and $\Gamma \times[-b, 0) \ni\left(x, t^{\prime}\right) \mapsto \Psi_{0}\left(x, t^{\prime}\right) \in \mathbb{R}$, respectively. 
The operator $\frac{\partial^{2}}{\partial t^{2}}+A$ in the state equation (6) is an infinite order hyperbolic operator and $A$ (Dubinskij, 1986; El-Saify and Bahaa, 2002) is given by

$$
A y=\left(\sum_{|\alpha|=0}^{\infty}(-1)^{|\alpha|} a_{\alpha} \mathcal{D}^{2 \alpha}+1\right) y
$$

and

$$
\sum_{|\alpha|=0}^{\infty}(-1)^{|\alpha|} a_{\alpha} \mathcal{D}^{2 \alpha}
$$

is an infinite order elliptic partial differential operator.

The operator $A$ is a mapping of $H^{\infty}\left\{a_{\alpha}, 2\right\}$ onto $H^{-\infty}\left\{a_{\alpha}, 2\right\}$. For this operator, the bilinear form $\Pi(t ; y, \varphi)=(A y, \varphi)_{L^{2}(\Omega)}$ is coercive on $H^{\infty}\left\{a_{\alpha}, 2\right\}$, i.e., there exists $\lambda>0, \lambda \in \mathbb{R}$ such that $\Pi(t ; y, \varphi) \geq$ $\lambda\|y\|_{H^{\infty}\left\{a_{\alpha}, 2\right\}}^{2}$. We assume that for any $y, \varphi \in$ $H^{\infty}\left\{a_{\alpha}, 2\right\}$ the function $t \rightarrow \Pi(t ; y, \varphi)$ is continuously differentiable in $[0, T]$ and $\Pi(t ; y, \varphi)=\Pi(t ; \varphi, y)$.

Equations (6)-111) constitute a Neumann problem. Define the right-hand side of (10) by

$$
q(x, t):=\int_{a}^{b} d(x, t) y(x, t-h) \mathrm{d} h+v(x, t) .
$$

Then (10) can be written as

$$
\begin{aligned}
\frac{\partial y}{\partial \eta_{A}}= & \sum_{|w|=0}^{\infty}\left(\mathcal{D}^{w} y(v)\right) \cos \left(n, x_{i}\right)=q(x, t) \\
& x \in \Gamma, t \in(0, T),
\end{aligned}
$$

where $\partial y / \partial \eta_{A}$ is the normal derivative at $\Gamma$, directed towards the exterior of $\Omega, \cos \left(n, x_{i}\right)$ is the $i$-th direction cosine of $n$, with $n$ being the normal at $\Gamma$ exterior to $\Omega$.

Remark 1. We shall apply the indication $q(x, t)$ appearing in (14) to prove the existence of a unique solution for (6)-11).

We shall formulate sufficient conditions for the existence of a unique solution of the mixed initial-boundary value problem (6)-111) for the cases where the function $u$ is a element of the space $H^{0,1}(Q)$ (i.e., $u \in$ $L^{2}\left(0, T ; H^{0}(\Omega)\right)=L^{2}(Q)$ and $u^{\prime}=\partial u / \partial t \in$ $\left.L^{2}\left(0, T ; H^{0}(\Omega)\right)\right)$.

We consider the Sobolev space $H^{\infty, 2}(Q)$ (Lions and Magenes, 1972) defined by

$$
\begin{array}{r}
H^{\infty, 2}(Q)=H^{0}\left(0, T ; H^{\infty}\left\{a_{\alpha}, 2\right\}(\Omega)\right) \\
\cap H^{2}\left(0, T ; H^{0}(\Omega)\right)
\end{array}
$$

which is a Hilbert space normed by

$$
\left.\left(\int_{0}^{T}\|y(t)\|_{H^{\infty}\left\{a_{\alpha}, 2\right\}(\Omega)}^{2} d t+\|y\|_{H^{2}\left(0, T ; H^{0}(\Omega)\right)}^{2}\right)^{1 / 2}\right)_{(16)}
$$

where $H^{2}\left(0, T ; H^{0}(\Omega)\right)$ denotes the Sobolev space of second order consisting of functions defined on $(0, T)$ and taking values in $H^{0}(\Omega)$.

For simplicity, we introduce the following notation:

$$
E_{j} \triangleq((j-1) a, j a), \quad Q_{j}=\Omega \times E_{j},
$$

$\Sigma_{j}=\Gamma \times E_{j} \quad$ for $j=1, \ldots, K$, where $K=T / a$.

The existence of a unique solution for the mixed initial-boundary value problem (6)-11) on the cylinder $Q$ can be proved using a constructive method, i.e., solving at first Eqns. (6) - (11) on the subcylinder $Q_{1}$ and in turn on $Q_{2}$, etc., until the procedure covers the whole cylinder $Q$. In this way, the solution in the previous step determines the next one.

Consequently, using Theorem 3.1 of (Lions and Magenes, 1972), one may prove the following lemma.

Lemma 1. Let

$$
\begin{gathered}
u \in H^{0,1}(Q), \\
f_{j} \in H^{0,1}\left(Q_{j}\right),
\end{gathered}
$$

where

$$
\begin{gathered}
f_{j}(x, t)=u(x, t)-\int_{a}^{b} c(x, t) y_{j-1}(x, t-h) \mathrm{d} h, \\
q_{j} \in H^{\infty, 3}\left(\Sigma_{j}\right),
\end{gathered}
$$

with

$$
\begin{gathered}
q_{j}(x, t)=\int_{a}^{b} d(x, t) y_{j-1}(x, t-h) \mathrm{d} h+v(x, t), \\
w_{j-1}(\cdot,(j-1) a) \\
=y_{j-1}(\cdot,(j-1) a) \in H^{\infty}\left\{a_{\alpha}, 2\right\}(\Omega), \\
w_{j-1}^{\prime}(\cdot,(j-1) a) \\
=y_{j-1}^{\prime}(\cdot,(j-1) a) \in H^{\infty}\left\{a_{\alpha}, 2\right\}(\Omega),
\end{gathered}
$$

and the following compatibility relations are fulfilled:

$$
\frac{\partial y_{j-1}}{\partial \eta_{A}}(x,(j-1) a)=q_{j}(x,(j-1) a) \quad \text { on } \Gamma,
$$




$$
\begin{aligned}
\frac{\partial y_{j-1}^{\prime}}{\partial \eta_{A}}(x, & (j-1) a) \\
& +\left(\frac{\partial}{\partial t}\left(\frac{\partial}{\partial \eta_{A}}\right)\right) y_{j-1}(x,(j-1) a) \\
= & \frac{\partial}{\partial t} q_{j}(x,(j-1) a) \text { on } \Gamma .
\end{aligned}
$$

Then, there exists a unique solution $y_{j} \in H^{\infty, 2}\left(Q_{j}\right)$ for the mixed initial-boundary value problem (6), (10), (20), (21).

Proof. For $j=1,\left.y_{0}\right|_{Q_{0}}(x, t-h)=\Phi_{0}(x, t-h)$ and $\left.y_{0}\right|_{\Sigma_{0}}(x, t-h)=\Psi_{0}(x, t-h)$, respectively. Then the assumptions (18)-(21) are fulfilled if $\Phi_{0} \in$ $H^{\infty, 2}\left(Q_{0}\right), v \in H^{\infty, 3}(\Sigma)$ and $\Psi_{0} \in H^{\infty, 3}\left(\Sigma_{0}\right)$. These assumptions are sufficient to ensure the existence of a unique solution $y_{1} \in H^{\infty, 2}\left(Q_{1}\right)$ if $y_{p} \in$ $H^{\infty}\left\{a_{\alpha}, 2\right\}(\Omega), y_{I} \in H^{\infty}\left\{a_{\alpha}, 2\right\}(\Omega)$ and the following compatibility conditions are satisfied:

$$
\begin{gathered}
\frac{\partial y_{p}}{\partial \eta_{A}}(x, 0)=q_{1}(x, 0) \text { on } \Gamma \\
\frac{\partial y_{I}}{\partial \eta_{A}}(x, 0)+\left(\frac{\partial}{\partial t}\left({\frac{\partial}{\partial \eta_{A}}}\right)\right) y_{p}(x, 0)=\frac{\partial}{\partial t} q_{1}(x, 0) \text { on } \Gamma
\end{gathered}
$$

In order to extend the result to $Q_{2}$, it is necessary to impose the compatibility relations

$$
\begin{gathered}
\frac{\partial y_{1}}{\partial \eta_{A}}(x, a)=q_{2}(x, a) \\
\frac{\partial y_{1}^{\prime}}{\partial \eta_{A}}(x, a)+\left(\frac{\partial}{\partial t}\left({\frac{\partial}{\partial \eta_{A}}}_{A}\right)\right) y_{1}(x, a)=\frac{\partial}{\partial t} q_{2}(x, a) \text { on } \Gamma
\end{gathered}
$$

and it is sufficient to verify that

$$
\begin{gathered}
f_{2} \in H^{0,1}\left(Q_{2}\right), \\
w_{1}(\cdot, a)=y_{1}(\cdot, a) \in H^{\infty}\left\{a_{\alpha}, 2\right\}(\Omega), \\
w_{1}^{\prime}(\cdot, a)=y_{1}^{\prime}(\cdot, a) \in H^{\infty}\left\{a_{\alpha}, 2\right\}(\Omega), \\
q_{2} \in H^{\infty, 3}\left(\Sigma_{2}\right) .
\end{gathered}
$$

First, using the solution in the previous step and the condition (17), we can immediately prove the condition (28).

To verify (29) and (30), we use the fact (by Proposition 3.1 of (Lions and Magenes, 1972)) that the function $w_{1}$ has the following properties:

$$
\begin{gathered}
w_{1} \in L^{2}\left(E_{1} ; H^{\infty}\left\{a_{\alpha}, 2\right\}(\Omega)\right), \\
w_{1}^{\prime} \in L^{2}\left(E_{1} ; H^{\infty}\left\{a_{\alpha}, 2\right\}(\Omega)\right), \\
w_{1}^{\prime \prime \prime} \in L^{2}\left(E_{1} ; H^{0}(\Omega)\right) .
\end{gathered}
$$

Then from Theorem 3.1 of (Lions and Magenes, 1972) it follows that the mappings $t \rightarrow w_{1}(\cdot, t)$ and $t \rightarrow w_{1}^{\prime}(\cdot, t)$ are continuous from $[0, a] \rightarrow H^{\infty}\left\{a_{\alpha}, 2\right\}(\Omega)$ and $[0, a] \rightarrow H^{\infty}\left\{a_{\alpha}, 2\right\}(\Omega)$, respectively. Hence $w_{1}(\cdot, a) \in H^{\infty}\left\{a_{\alpha}, 2\right\}(\Omega)$ and $w_{1}^{\prime}(\cdot, a) \in H^{\infty}\left\{a_{\alpha}, 2\right\}(\Omega)$. But from Section 3 of (Lions and Magenes, 1972) it follows that $w_{1}(\cdot, a)=y_{1}(\cdot, a)$ and $w_{1}^{\prime}(\cdot, a)=y_{1}^{\prime}(\cdot, a)$. From the proceding results we can deduce that $y_{1}(\cdot, a) \in H^{\infty}\left\{a_{\alpha}, 2\right\}(\Omega)$ and $y_{1}^{\prime}(\cdot, a) \in H^{\infty}\left\{a_{\alpha}, 2\right\}(\Omega)$. Again, from the trace theorem of (Lions and Magenes, 1972), $y_{1} \in H^{\infty, 2}\left(Q_{1}\right)$ implies that $\left.y_{1} \rightarrow y_{1}\right|_{\Sigma_{1}}$ is a linear continuous mapping of $H^{\infty, 2}\left(Q_{1}\right) \rightarrow H^{\infty, 2}\left(\sum_{1}\right) \subset H^{\infty, 3}\left(\sum_{1}\right)$. Thus $\left.y_{1}\right|_{\sum_{1}} \in H^{\infty, 3}\left(\Sigma_{1}\right)$. Assuming that $c$ is a $C^{\infty}$ function and $v \in H^{\infty, 3}(\Sigma)$, the condition (31) is fulfilled. Then, there exists a unique solution $y_{2} \in H^{\infty, 2}\left(Q_{2}\right)$. We shall now extend our result to any $Q_{j}, 2<j \leq K$.

Theorem 1. Let $y_{p}, y_{I}, \Phi_{0}, \Psi_{0}, v$ and $u$ be given with $y_{p} \in H^{\infty}\left\{a_{\alpha}, 2\right\}(\Omega), y_{I} \in H^{\infty}\left\{a_{\alpha}, 2\right\}(\Omega)$, $\Phi_{0} \in H^{\infty, 2}\left(Q_{0}\right), \Psi_{0} \in H^{\infty, 3}\left(\Sigma_{0}\right), v \in H^{\infty, 3}\left(\sum\right)$, $u \in H^{0,1}(Q)$ and the compatibility relations (24), (25) be fulfilled. Then there exists a unique solution $y \in$ $H^{\infty, 2}(Q)$ for the mixed initial-boundary value problem (6)-(11) with $y(\cdot, a) \in H^{\infty}\left\{a_{\alpha}, 2\right\}(\Omega)$ and $y^{\prime}(\cdot, a) \in$ $H^{\infty}\left\{a_{\alpha}, 2\right\}(\Omega)$ for $j=1, \ldots, K$.

\section{Problem formulation. Optimization theo- rems}

Now, we shall formulate the minimum-time problem for (6) - 111) in the context of Theorem 1 that is,

$$
u \in U_{Q_{\mathrm{ad}}}=\left\{u \in H^{0,1}(Q):|u(x, t)| \leq 1\right\} .
$$

We shall define the target set $W$ such that

$$
W=\left\{y \in L^{2}(\Omega):\left\|y-z_{d}\right\|_{L^{2}(\Omega)} \leq \varepsilon\right\},
$$

where $z_{d}, \varepsilon$ are given with $z_{d} \in L^{2}(\Omega)$ and $\varepsilon>0$.

The solving of the formulated minimum-time problem is equivalent to hitting the target set $W$ in minimum time.

Moreover, we assume that

$$
\begin{aligned}
& \text { there exists a } T>0 \text { and } u \in U_{Q_{\mathrm{ad}}} \\
& \text { with }\left(y(T ; u), \frac{\partial y(T ; u)}{\partial t}\right) \in W .
\end{aligned}
$$

Theorem 2. If the assumption (34) holds, then the set $W$ is reached in minimum time $t^{*}$ by an admissible control $u^{*} \in U_{Q_{\mathrm{ad}}}$. Moreover,

$$
\begin{array}{r}
-\int_{\Omega}\left(z_{d}-y\left(t^{*} ; u^{*}\right)\right)\left(y\left(t^{*} ; u\right)-y\left(t^{*} ; u^{*}\right)\right) \mathrm{d} x \leq 0, \\
\forall u \in U_{Q_{\mathrm{ad}}},
\end{array}
$$


Proof. Let us define the following set:

$$
\begin{array}{r}
t^{*} \stackrel{d f}{=} \inf \left\{t: \quad\left(y(t ; u), \frac{\partial y(t ; u)}{\partial t}\right) \in W\right. \\
\text { for some } \left.u \in U_{Q_{\mathrm{ad}}}\right\} .
\end{array}
$$

The infimum is well defined, as (34) quarantees that this set is nonempty. By definition, we can choose $t_{n} \downarrow t^{*}$ and admissible controls $\left\{u_{n}\right\}$ such that

$$
\left(y\left(t_{n} ; u_{n}\right), \frac{\partial y\left(t_{n} ; u_{n}\right)}{\partial t}\right) \in W, n=1,2,3, \ldots
$$

Each $u_{n}$ is defined on $\Omega \times\left(0, t_{n}\right) \supset \Omega \times\left(0, t^{*}\right)$. To simplify the notation, we denote the restriction of $u_{n}$ to $\Omega \times$ $\left(0, t^{*}\right)$ again by $u_{n}$. The set of admissible controls then forms a weakly compact, convex set in $H^{0,1}\left(\Omega \times\left(0, t^{*}\right)\right)$, and so we can extract a weakly convergent subset $\left\{u_{m}\right\}$, which converges weakly to some admissible control $u^{*}$.

Consequently, Theorem 1 implies that $y(t ; u) \in H^{\infty}\left\{a_{\alpha}, 2\right\}(\Omega) \subset L^{2}(\Omega)$ and $\partial y(t ; u) / \partial t$ $\in H^{\infty}\left\{a_{\alpha}, 2\right\}(\Omega) \subset L^{2}(\Omega)$ for each $u \in H^{0,1}(Q)$ and $t>0$. Then, using Theorem 1.1 of (Lions, 1971) and Theorem 1, we can prove that the mapping $H^{0,1}\left(\Omega \times\left(0, t^{*}\right)\right) \rightarrow L^{2}(\Omega) \times L^{2}(\Omega)$, defined by

$$
u \mapsto\left(y\left(t^{*} ; u\right), \frac{\partial y\left(t^{*} ; u\right)}{\partial t}\right),
$$

is continuous. Since any continuous linear mapping between Banach spaces is also weakly continuous (Dunford and Schwartz, 1958, Theorem V. 3.15), the affine mapping

$$
u \mapsto\left(y\left(t^{*} ; u\right), \frac{\partial y\left(t^{*} ; u\right)}{\partial t}\right)
$$

must also be weakly continuous. Hence

$$
\left\{\begin{array}{l}
y\left(t^{*} ; u_{m}\right) \rightarrow y\left(t^{*} ; u^{*}\right) \text { weakly in } L^{2}(\Omega), \\
\dot{y}\left(t^{*} ; u_{m}\right) \rightarrow \dot{y}\left(t^{*} ; u^{*}\right) \text { weakly in } L^{2}(\Omega) .
\end{array}\right.
$$

Further,

$$
\begin{gathered}
\frac{\partial y(u)}{\partial t} \in L^{2}\left(\left[0, t^{*}\right], H^{0}(\Omega)\right), \\
\frac{\partial^{2} y(u)}{\partial t^{2}} \in L^{2}\left(\left[0, t^{*}\right], H^{0}(\Omega)\right),
\end{gathered}
$$

for each $u \in U$, by definition of $H^{\infty, 2}\left(\Omega \times\left(0, t^{*}\right)\right)$, and

$$
\begin{aligned}
& \left\|y\left(t_{m} ; u_{m}\right)-y\left(t^{*} ; u_{m}\right)\right\|_{L^{2}(\Omega)} \\
& =\left\|\int_{t^{*}}^{t_{m}} \dot{y}\left(\sigma ; u_{m}\right) \mathrm{d} \sigma\right\|_{L^{2}(\Omega)} \\
& \leq \sqrt{t_{m}-t^{*}}\left(\int_{t^{*}}^{t_{m}}\left\|\dot{y}\left(\sigma ; u_{m}\right)\right\|_{L^{2}(\Omega)}^{2} \mathrm{~d} \sigma\right)^{1 / 2},
\end{aligned}
$$

$$
\begin{aligned}
& \left\|\dot{y}\left(t_{m} ; u_{m}\right)-\dot{y}\left(t^{*} ; u_{m}\right)\right\|_{L^{2}(\Omega)} \\
& \quad=\left\|\int_{t^{*}}^{t_{m}} \ddot{y}\left(\sigma ; u_{m}\right) \mathrm{d} \sigma\right\|_{L^{2}(\Omega)} \\
& \quad \leq \sqrt{t_{m}-t^{*}}\left(\int_{t^{*}}^{t_{m}}\left\|\ddot{y}\left(\sigma ; u_{m}\right)\right\|_{L^{2}(\Omega)}^{2} \mathrm{~d} \sigma\right)^{1 / 2} .
\end{aligned}
$$

Applying Theorem 1.1 of (Lions, 1971) and Theorem 1 again, the sets $\left\{\dot{y}\left(u_{m}\right)\right\}$ and $\left\{\ddot{y}\left(u_{m}\right)\right\}$ must be bounded in $L^{2}\left(0, t^{*} ; H^{0}(\Omega)\right)$, and so

$$
\left\{\begin{array}{l}
\left\|y\left(t_{m} ; u_{m}\right)-y\left(t^{*} ; u_{m}\right)\right\|_{L^{2}(\Omega)} \leq M \sqrt{t_{m}-t^{*}} \\
\left\|\dot{y}\left(t_{m} ; u_{m}\right)-\dot{y}\left(t^{*} ; u_{m}\right)\right\|_{L^{2}(\Omega)} \leq M_{1} \sqrt{t_{m}-t^{*}} .
\end{array}\right.
$$

Combining (38) and (41) shows that

$$
\left\{\begin{array}{l}
y\left(t_{m} ; u_{m}\right)-y\left(t^{*} ; u^{*}\right) \\
=\left(y\left(t_{m} ; u_{m}\right)-y\left(t^{*} ; u_{m}\right)\right) \\
\quad+\left(y\left(t^{*} ; u_{m}\right)-y\left(t^{*} ; u^{*}\right)\right) \\
\dot{y}\left(t_{m} ; u_{m}\right)-\dot{y}\left(t^{*} ; u^{*}\right) \\
=\left(\dot{y}\left(t_{m} ; u_{m}\right)-\dot{y}\left(t^{*} ; u_{m}\right)\right) \\
\quad+\left(\dot{y}\left(t^{*} ; u_{m}\right)-\dot{y}\left(t^{*} ; u^{*}\right)\right)
\end{array}\right.
$$

converges weakly to zero in $L^{2}(\Omega)$, and so

$$
\left(y\left(t^{*} ; u^{*}\right), \frac{\partial y\left(t^{*} ; u^{*}\right)}{\partial t}\right) \in W
$$

as $W$ is closed and convex, hence weakly closed. This shows that $W$ is reached in time $t^{*}$ by an admissible control accordingly, $t^{*}$ must be the minimum time and $u^{*}$ an optimal control.

We shall now prove the second part of our theorem. Indeed, from Theorem 3.1 of (Lions and Magenes, 1972), $y(u) \in H^{\infty, 2}(Q)$ implies that the mappings $t \rightarrow y(t ; u)$ and $t \rightarrow y^{\prime}(t ; u)$ from $[0, T] \rightarrow H^{\infty}\left\{a_{\alpha}, 2\right\}(\Omega) \subset L^{2}(\Omega)$ and $[0, T] \rightarrow H^{\infty}\left\{a_{\alpha}, 2\right\}(\Omega) \subset L^{2}(\Omega)$ are continuous for each fixed $u$, and so

$$
\left(y\left(t^{*} ; u\right) ; \frac{\partial y\left(t^{*} ; u\right)}{\partial t}\right) \notin \operatorname{int} W,
$$

for any $u \in U_{Q_{\mathrm{ad}}}$, by the minimality of $t^{*}$.

From our earlier remarks, the set

$$
\mathcal{A}\left(t^{*}\right)=\left\{y\left(t^{*} ; u_{x}\right): u_{x} \in U_{Q_{\mathrm{ad}}}\right\}
$$

is a continuous affine image of the weakly compact and convex in $L^{2}(\Omega)$. Applying Theorem 21.11 of (Choquet, $1969)$ to the sets $\mathcal{A}\left(t^{*}\right)$ and $W$ shows that there exists a nontrivial hyperplane $z^{\prime} \in L^{2}(\Omega)$ separating these sets, that is,

$$
-\int_{\Omega} z^{\prime} y\left(t^{*} ; u\right) \mathrm{d} x \leq-\int_{\Omega} z^{\prime} y\left(t^{*} ; u^{*}\right) \mathrm{d} x \leq-\int_{\Omega} z^{\prime} y \mathrm{~d} x
$$


for all $u \in U_{Q_{a d}}$ and $y \in L^{2}(\Omega)$ with

$$
\left\|y-z_{d}\right\|_{L^{2}(\Omega)} \leq \varepsilon .
$$

From the second inequality in (44), $z^{\prime}$ must support the set $W$ at

$$
\left(y\left(t^{*} ; u^{*}\right), \frac{\partial y\left(t^{*} ; u^{*}\right)}{\partial t}\right)
$$

and, since $L^{2}(\Omega)$ is a Hilbert space, $z^{\prime}$ must be of the form

$$
z^{\prime}=\lambda\left(z_{d}-y\left(t^{*} ; u^{*}\right)\right) \text { for some } \lambda>0 .
$$

Subsequently, dividing (44) by $\lambda$ gives the desired result (35).

We shall apply Theorem 2 to the control problem of (6) - (11)

To simplify (35), we introduce the adjoint equation and for every $u \in U_{Q_{a d}}$ we define the adjoint variable $p=p(u)=p(x, t ; u)$ as the solution of the equation

$$
\begin{aligned}
& \frac{\partial^{2} p(u)}{\partial t^{2}}+A p(u) \\
& \quad+\int_{a}^{b} c(x, t+h) p(x, t+h ; u) \mathrm{d} h=0, \\
& \quad x \in \Omega, \quad t \in\left(0, t^{*}-b\right),
\end{aligned}
$$

$$
\begin{gathered}
\frac{\partial^{2} p(u)}{\partial t^{2}}+A p(u) \\
+\int_{a}^{t^{*}-t} c(x, t+h) p(x, t+h ; u) \mathrm{d} h=0, \\
\quad x \in \Omega, \quad t \in\left(t^{*}-b, t^{*}-a\right), \\
\frac{\partial^{2} p(u)}{\partial t^{2}}+A p(u)=0, \quad x \in \Omega, \quad t \in\left(t^{*}-a, t^{*}\right), \\
p^{\prime}\left(x, t^{*} ; u\right)=z_{d}(x)-y\left(x, t^{*} ; u\right), \quad x \in \Omega, \\
\frac{\partial p(u)}{\partial \eta_{A}}(x, t)=\int_{a} d(x, t+h) p(x, t+h ; u) \mathrm{d} h, \\
\frac{\partial p(u)}{\partial \eta_{A}}(x, t)=\int_{a}^{t^{*}-t} d(x, t+h) p(x, t+h ; u) \mathrm{d} h, \quad t \in\left(0, t^{*}-b\right), \\
\frac{\partial p(u)}{\partial \eta_{A}}(x, t)=0, \quad x \in \Gamma, \quad t \in\left(t^{*}-a, t^{*}\right),
\end{gathered}
$$

where

$$
\left.\begin{array}{l}
\frac{\partial p(u)}{\partial \eta_{A}}(x, t)=\sum_{|w|=0}^{\infty}\left(\mathcal{D}^{w} p(u)\right) \cos \left(n, x_{i}\right) \\
A p=\left(\sum_{|\alpha|=0}^{\infty}(-1)^{|\alpha|} a_{\alpha} \mathcal{D}^{2 \alpha}+1\right) p
\end{array}\right\} .
$$

Remark 2. If $t^{*}<b$, then we consider (48), (53) on $\Omega \times\left(0, t^{*}-a\right)$ and $\Gamma \times\left(0, t^{*}-a\right)$, respectively.

The existence of a unique solution for the problem (47) - 54) on the cylinder $\Omega \times\left(0, t^{*}\right)$ can be proved using a constructive method. It is easy to notice that, for given $z_{d}$ and $u$, the problem (47)-(54) can be solved backwards in time starting from $t=t^{*}$, i.e., first, solving (47)-(54) on the subcylinder $Q_{K}$ and in turn on $Q_{K-1}$, etc., until the procedure covers the whole cylinder $\Omega \times\left(0, t^{*}\right)$. For this purpose, we may apply Theorem 1 (with an obvious change of variables). result.

Hence, using Theorem 1 , one can prove the following

Lemma 2. Let the hypothesis of Theorem 1 be satisfied. Given $z_{d} \in L^{2}(\Omega)$ and any $u \in H^{0,1}(Q)$, there exists a unique solution $p(u) \in H^{\infty, 2}\left(\Omega \times\left(0, t^{*}\right)\right)$ for the adjoint problem (47)-(54).

We simplify (35) using the adjoint equations 47) (54). For this purpose, setting $u=u^{*}$ in (47)-(54), multiplying both sides of (47), (48) and (49) by $y(u)-y\left(u^{*}\right)$, then integrating over $\Omega \times\left(0, t^{*}-b\right), \Omega \times\left(t^{*}-b, t^{*}-a\right)$ and $\Omega \times\left(t^{*}-a, t^{*}\right)$, respectively, and then adding both sides of 47, (48) and 49), we get

$$
\begin{aligned}
\int_{0} \int_{\Omega}\left(\frac{\partial^{2} p\left(u^{*}\right)}{\partial t^{2}}+A p\left(u^{*}\right)\right)\left(y(u)-y\left(u^{*}\right)\right) \mathrm{d} x \mathrm{~d} t \\
+\int_{0} \int_{\Omega}\left(\int_{a}^{b} c(x, t+h) p\left(x, t+h ; u^{*}\right) \mathrm{d} h\right) \\
\quad \times\left[y(x, t ; u)-y\left(x, t ; u^{*}\right)\right] \mathrm{d} x \mathrm{~d} t \\
+\int_{t^{*}-a} \int_{\Omega}\left(\int_{a}^{t^{*}-t} c(x, t+h) p\left(x, t+h ; u^{*}\right) \mathrm{d} h\right) \\
\quad \times\left[y(x, t ; u)-y\left(x, t ; u^{*}\right)\right] \mathrm{d} x \mathrm{~d} t \\
\quad \int_{\Omega} p^{\prime}\left(x, t^{*} ; u^{*}\right)\left(y\left(x, t^{*} ; u\right)-y\left(x, t^{*} ; u^{*}\right)\right) \mathrm{d} x \\
+\int_{0}^{t^{*}} \int_{\Omega} p\left(u^{*}\right) \frac{\partial^{2}}{\partial t^{2}}\left(y(u)-y\left(u^{*}\right)\right) \mathrm{d} x \mathrm{~d} t \\
+\int_{0}^{t^{*}} \int_{\Omega} A^{*} p\left(u^{*}\right)\left(y(u)-y\left(u^{*}\right)\right) \mathrm{d} x \mathrm{~d} t \\
+\int_{0}^{t^{*}-b} \int_{\Omega} \int_{a}^{b} c(x, t+h) p\left(x, t+h ; u^{*}\right) \\
\left.\quad \times(x, t u) y\left(x, t ; u^{*}\right)\right) \mathrm{d} h \mathrm{~d} x \mathrm{~d} t
\end{aligned}
$$




$$
\begin{aligned}
& +\int_{t^{*}-b}^{t^{*}-a} \int_{\Omega} \int_{a}^{t^{*}-t} c(x, t+h) p\left(x, t+h ; u^{*}\right) \\
& \quad \times\left(y(x, t ; u)-y\left(x, t ; u^{*}\right)\right) \mathrm{d} h \mathrm{~d} x \mathrm{~d} t=0 .
\end{aligned}
$$

Then applying (51), the formula (56) can be expressed as

$$
\begin{aligned}
-\int_{\Omega}\left(z_{d}-y\left(t^{*} ; u^{*}\right)\right)\left(y\left(t^{*} ; u\right)-y\left(t^{*} ; u^{*}\right)\right) \mathrm{d} x \\
=\int_{0} \int_{\Omega} p\left(u^{*}\right) \frac{\partial^{2}}{\partial t^{2}}\left(y(u)-y\left(u^{*}\right)\right) \mathrm{d} x \mathrm{~d} t \\
+\int_{0}^{t_{\Omega}^{*}} \int_{\Omega} A p\left(u^{*}\right)\left(y(u)-y\left(u^{*}\right)\right) \mathrm{d} x \mathrm{~d} t \\
+\int_{a}^{b} \int_{\Omega}^{t^{*}-b} c(x, t+h) p\left(x, t+h ; u^{*}\right) \\
\quad \times\left(y(x, t ; u)-y\left(x, t ; u^{*}\right)\right) \mathrm{d} t \mathrm{~d} x \mathrm{~d} h \\
+\int_{a}^{t^{*}-t} \int_{\Omega}^{t^{*}-a} c(x, t+h) p\left(x, t+h ; u^{*}\right) \\
\quad \times\left(y(x, t ; u)-y\left(x, t ; u^{*}\right)\right) \mathrm{d} t \mathrm{~d} x \mathrm{~d} h .
\end{aligned}
$$

Using Eqn. (6), the first integral on the right-hand side of (57) can be rewritten as

$$
\begin{gathered}
\int_{0}^{t_{\Omega}^{*}} \int_{0} p\left(u^{*}\right) \frac{\partial}{\partial t}\left(y(u)-y\left(u^{*}\right)\right) \mathrm{d} x \mathrm{~d} t \\
=\int_{0}^{t^{*}} \int_{\Omega} p\left(u^{*}\right)\left(u-u^{*}\right) \mathrm{d} x \mathrm{~d} t \\
-\int_{0}^{t^{*}} \int_{\Omega} p\left(u^{*}\right) A\left(y(u)-y\left(u^{*}\right)\right) \mathrm{d} x \mathrm{~d} t, \\
\int_{\Omega}^{t^{*} p\left(x, t ; u^{*}\right)} \int_{a} c(x, t) \\
\left.\times\left(y(x, t-h ; u)-y\left(x, t-h ; u^{*}\right)\right) \mathrm{d} h\right) \mathrm{d} x \mathrm{~d} t \\
=\int_{0}^{t^{*}} \int_{\Omega} p\left(u^{*}\right)\left(u-u^{*}\right) \mathrm{d} x \mathrm{~d} t \\
-\int_{0} \int_{\Omega} p\left(u^{*}\right) A\left(y(u)-y\left(u^{*}\right)\right) \mathrm{d} x \mathrm{~d} t
\end{gathered}
$$

$$
\begin{aligned}
& -\int_{0}^{t^{*}} \int_{\Omega} \int_{a}^{b} p\left(x, t ; u^{*}\right) c(x, t) \\
& \quad \times\left(y(x, t-h ; u)-y\left(x, t-h ; u^{*}\right)\right) \mathrm{d} h \mathrm{~d} x \mathrm{~d} t
\end{aligned}
$$$$
=\int_{0}^{t^{*}} \int_{\Omega} p\left(u^{*}\right)\left(u-u^{*}\right) \mathrm{d} x \mathrm{~d} t
$$$$
-\int_{0}^{t^{*}} \int_{\Omega} p\left(u^{*}\right) A\left(y(u)-y\left(u^{*}\right)\right) \mathrm{d} x \mathrm{~d} t,
$$$$
-\int_{a}^{b} \int_{\Omega} \int_{0}^{t^{*}} p\left(x, t ; u^{*}\right) c(x, t)
$$$$
\times\left(y(x, t-h ; u)-y\left(x, t-h ; u^{*}\right)\right) \mathrm{d} t \mathrm{~d} x \mathrm{~d} h
$$$$
=\int_{0}^{t^{*}} \int_{\Omega} p\left(u^{*}\right)\left(u-u^{*}\right) \mathrm{d} x \mathrm{~d} t
$$$$
-\int_{0}^{t^{*}} \int_{\Omega} p\left(u^{*}\right) A\left(y(u)-y\left(u^{*}\right)\right) \mathrm{d} x \mathrm{~d} t
$$$$
-\int_{a}^{b} \int_{\Omega}^{t^{*}-h} \int_{-h}^{h} p\left(x, t^{\prime}+h ; u^{*}\right) c\left(x, t^{\prime}+h\right)
$$$$
\times\left(y\left(x, t^{\prime} ; u\right)-y\left(x, t^{\prime} ; u^{*}\right)\right) \mathrm{d} t^{\prime} \mathrm{d} x \mathrm{~d} h
$$$$
=\int_{0}^{t^{*}} \int_{\Omega} p\left(u^{*}\right)\left(u-u^{*}\right) \mathrm{d} x \mathrm{~d} t
$$$$
-\int_{0}^{t^{*}} \int_{\Omega} p\left(u^{*}\right) A\left(y(u)-y\left(u^{*}\right)\right) \mathrm{d} x \mathrm{~d} t
$$$$
-\int_{a}^{b} \int_{\Omega} \int_{-h}^{0} p\left(x, t^{\prime}+h ; u^{*}\right) c\left(x, t^{\prime}+h\right)
$$$$
\times\left(y\left(x, t^{\prime} ; u\right)-y\left(x, t^{\prime} ; u^{*}\right)\right) \mathrm{d} t^{\prime} \mathrm{d} x \mathrm{~d} h
$$$$
-\int_{a}^{b} \int_{\Omega}^{t^{*}-b} \int_{0} p\left(x, t^{\prime}+h ; u^{*}\right) c\left(x, t^{\prime}+h\right)
$$$$
\times\left(y\left(x, t^{\prime} ; u\right)-y\left(x, t^{\prime} ; u^{*}\right)\right) \mathrm{d} t^{\prime} \mathrm{d} x \mathrm{~d} h
$$$$
-\int_{a}^{b} \int_{\Omega} \int_{t^{*}-b}^{t^{*}-h} p\left(x, t^{\prime}+h ; u^{*}\right) c\left(x, t^{\prime}+h\right)
$$$$
\times\left(y\left(x, t^{\prime} ; u\right)-y\left(x, t^{\prime} ; u^{*}\right)\right) \mathrm{d} t^{\prime} \mathrm{d} x \mathrm{~d} h
$$

$=\int_{0}^{t^{*}} \int_{\Omega} p\left(u^{*}\right)\left(u-u^{*}\right) \mathrm{d} x \mathrm{~d} t$ 


$$
\begin{aligned}
& -\int_{0} \int_{\Omega} p\left(u^{*}\right) A\left(y(u)-y\left(u^{*}\right)\right) \mathrm{d} x \mathrm{~d} t \\
& -\int_{a}^{b} \int_{\Omega} \int_{-h}^{0} p\left(x, t^{\prime}+h ; u^{*}\right) c\left(x, t^{\prime}+h\right) \\
& \quad \times\left(y\left(x, t^{\prime} ; u\right)-y\left(x, t^{\prime} ; u^{*}\right)\right) \mathrm{d} t^{\prime} \mathrm{d} x \mathrm{~d} h \\
& -\int_{a}^{b} \int_{\Omega}^{t_{0}^{*}-b} p\left(x, t^{\prime}+h ; u^{*}\right) c\left(x, t^{\prime}+h\right) \\
& \quad \times\left(y\left(x, t^{\prime} ; u\right)-y\left(x, t^{\prime} ; u^{*}\right)\right) \mathrm{d} t^{\prime} \mathrm{d} x \mathrm{~d} h \\
& \quad \int^{*}-t \int_{a}^{t^{*}-a} p\left(x, t^{\prime}+h ; u^{*}\right) c\left(x, t^{\prime}+h\right) \\
& \quad \times\left(y\left(x, t^{\prime} ; u\right)-y\left(x, t^{\prime} ; u^{*}\right)\right) \mathrm{d} t^{\prime} \mathrm{d} x \mathrm{~d} h .
\end{aligned}
$$

The second integral on the right-hand side of 57), in view of Green's formula, can be expressed as

$$
\begin{aligned}
& \int_{0}^{t^{*}} \int_{\Omega} A p\left(u^{*}\right)\left(y(u)-y\left(u^{*}\right)\right) \mathrm{d} x \mathrm{~d} t \\
& =\int_{0}^{t^{*}} \int_{\Omega} p\left(u^{*}\right) A\left(y(u)-y\left(u^{*}\right)\right) \mathrm{d} x \mathrm{~d} t \\
& +\int_{0}^{t_{\Gamma}^{*}} p\left(u^{*}\right)\left(\frac{\partial y(u)}{\partial \eta_{A}}-\frac{\partial y\left(u^{*}\right)}{\partial \eta_{A}}\right) \mathrm{d} \Gamma \mathrm{d} t \\
& -\int_{0}^{t^{*}} \int_{\Gamma} \frac{\partial p\left(u^{*}\right)}{\partial \eta_{A^{*}}}\left(y(u)-y\left(u^{*}\right)\right) \mathrm{d} \Gamma \mathrm{d} t .
\end{aligned}
$$

Using the boundary condition (10), the second component on the right-hand side of (59) can be written as

$$
\begin{aligned}
\int_{0}^{t^{*}} \int_{\Gamma} p\left(u^{*}\right) & {\left[\frac{\partial y(u)}{\partial \eta_{A}}-\frac{\partial y\left(u^{*}\right)}{\partial \eta_{A}}\right] \mathrm{d} \Gamma \mathrm{d} t } \\
= & \int_{0}^{t^{*}} \int_{\Gamma} p\left(x, t ; u^{*}\right)\left(\int_{a}^{b} d(x, t)\right. \\
& \left.\times\left(y(x, t-h ; u)-y\left(x, t-h ; u^{*}\right)\right) \mathrm{d} h\right) \mathrm{d} \Gamma \mathrm{d} t \\
= & \int_{0}^{t_{\Gamma}^{*}} \int_{a} \int_{a}^{b} p\left(x, t ; u^{*}\right) d(x, t) \\
& \times\left(y(x, t-h ; u)-y\left(x, t-h ; u^{*}\right)\right) \mathrm{d} h \mathrm{~d} \Gamma \mathrm{d} t
\end{aligned}
$$

$$
\begin{aligned}
& =\int_{a}^{b} \int_{\Gamma} \int_{0}^{t^{*}} p\left(x, t ; u^{*}\right) \mathrm{d}(x, t) \\
& \times\left(y(x, t-h ; u)-y\left(x, t-h ; u^{*}\right)\right) \mathrm{d} t \mathrm{~d} \Gamma \mathrm{d} h \\
& =\int_{a}^{b} \int_{\Gamma}^{t^{*}} \int_{-h}^{-h} p\left(x, t^{\prime}+h ; u^{*}\right) d\left(x, t^{\prime}+h\right) \\
& \times\left(y\left(x, t^{\prime} ; u\right)-y\left(x, t^{\prime} ; u^{*}\right)\right) \mathrm{d} t^{\prime} \mathrm{d} \Gamma \mathrm{d} h \\
& =\int_{a}^{b} \int_{\Gamma} \int_{-h}^{0} p\left(x, t^{\prime}+h ; u^{*}\right) d\left(x, t^{\prime}+h\right) \\
& \times\left(y\left(x, t^{\prime} ; u\right)-y\left(x, t^{\prime} ; u^{*}\right)\right) \mathrm{d} t^{\prime} \mathrm{d} \Gamma \mathrm{d} h \\
& +\int_{a}^{b} \int_{\Gamma}^{t^{*}} \int_{0}^{-b} p\left(x, t^{\prime}+h ; u^{*}\right) d\left(x, t^{\prime}+h\right) \\
& \times\left(y\left(x, t^{\prime} ; u\right)-y\left(x, t^{\prime} ; u^{*}\right)\right) \mathrm{d} t^{\prime} \mathrm{d} \Gamma \mathrm{d} h \\
& +\int_{a}^{b} \int_{\Gamma} \int_{t^{*}-b}^{t^{*}-h} p\left(x, t^{\prime}+h ; u^{*}\right) d\left(x, t^{\prime}+h\right) \\
& \times\left(y\left(x, t^{\prime} ; u\right)-y\left(x, t^{\prime} ; u^{*}\right)\right) \mathrm{d} t^{\prime} \mathrm{d} \Gamma \mathrm{d} h \\
& =\int_{a}^{b} \int_{\Gamma} \int_{-h}^{0} p\left(x, t^{\prime}+h ; u^{*}\right) d\left(x, t^{\prime}+h\right) \\
& \times\left(y\left(x, t^{\prime} ; u\right)-y\left(x, t^{\prime} ; u^{*}\right)\right) \mathrm{d} t^{\prime} \mathrm{d} \Gamma \mathrm{d} h \\
& +\int_{a}^{b} \int_{\Gamma}^{t^{*}-b} \int_{0}^{b} p\left(x, t^{\prime}+h ; u^{*}\right) d\left(x, t^{\prime}+h\right) \\
& \times\left(y\left(x, t^{\prime} ; u\right)-y\left(x, t^{\prime} ; u^{*}\right)\right) \mathrm{d} t^{\prime} \mathrm{d} \Gamma \mathrm{d} h \\
& +\int_{a}^{t^{*}-t} \int_{\Gamma} \int_{t^{*}-b}^{t^{*}-a} p\left(x, t^{\prime}+h ; u^{*}\right) d\left(x, t^{\prime}+h\right) \\
& \times\left(y\left(x, t^{\prime} ; u\right)-y\left(x, t^{\prime} ; u^{*}\right)\right) \mathrm{d} t^{\prime} \mathrm{d} \Gamma \mathrm{d} h .
\end{aligned}
$$

The last component in (59) can be rewritten as

$$
\begin{aligned}
& \int_{0}^{t^{*}} \int_{\Gamma} \frac{\partial p\left(u^{*}\right)}{\partial \eta_{A^{*}}}\left(y(u)-y\left(u^{*}\right)\right) \mathrm{d} \Gamma \mathrm{d} t \\
& =\int_{0}^{t^{*}-b} \int_{\Gamma} \frac{\partial p\left(u^{*}\right)}{\partial \eta_{A^{*}}}\left(y(u)-y\left(u^{*}\right)\right) \mathrm{d} \Gamma \mathrm{d} t \\
& +\int_{t^{*}-b}^{t^{*}-a} \frac{\partial p\left(u^{*}\right)}{\partial \eta_{A^{*}}}\left(y(u)-y\left(u^{*}\right)\right) \mathrm{d} \Gamma \mathrm{d} t \\
& +\int_{t^{*}-a}^{t^{*}} \frac{\partial p\left(u^{*}\right)}{\partial \eta_{A^{*}}}\left(y(u)-y\left(u^{*}\right)\right) \mathrm{d} \Gamma \mathrm{d} t .
\end{aligned}
$$


Substituting (60), (61) into (59) and then (58), (59) into (57), we obtain

$$
\begin{aligned}
& -\int_{\Omega}\left(z_{d}-y\left(t^{*} ; u^{*}\right)\right)\left(y\left(t^{*} ; u\right)-y\left(t^{*} ; u^{*}\right)\right) \mathrm{d} x \\
& =\int_{0}^{t^{*}} \int_{\Omega} p\left(u^{*}\right)\left(u-u^{*}\right) \mathrm{d} x \mathrm{~d} t \\
& =-\int_{0}^{t^{*}} \int_{\Omega} p\left(u^{*}\right) A\left(y(u)-y\left(u^{*}\right)\right) \mathrm{d} x \mathrm{~d} t \\
& -\int_{a}^{b} \int_{\Omega} \int_{-h}^{0} c(x, t+h) p\left(x, t+h ; u^{*}\right) \\
& \times\left(y(x, t ; u)-y\left(x, t ; u^{*}\right)\right) \mathrm{d} t \mathrm{~d} x \mathrm{~d} h \\
& -\int_{a}^{b} \int_{\Omega}^{t^{*}} \int_{0}^{-b} c(x, t+h) p\left(x, t+h ; u^{*}\right) \\
& \times\left(y(x, t ; u)-y\left(x, t ; u^{*}\right)\right) \mathrm{d} t \mathrm{~d} x \mathrm{~d} h \\
& -\int_{a}^{t^{*}-t} \int_{\Omega} \int_{t^{*}-b}^{t^{*}-a} c(x, t+h) p\left(x, t+h ; u^{*}\right) \\
& \times\left(y(x, t ; u)-y\left(x, t ; u^{*}\right)\right) \mathrm{d} t \mathrm{~d} x \mathrm{~d} h \\
& +\int_{0}^{t^{*}} \int_{\Omega} p\left(u^{*}\right) A\left(y(u)-y\left(u^{*}\right)\right) \mathrm{d} x \mathrm{~d} t \\
& +\int_{a}^{b} \int_{\Gamma} \int_{-h}^{0} d(x, t+h) p\left(x, t+h ; u^{*}\right) \\
& \times\left(y(x, t ; u)-y\left(x, t ; u^{*}\right)\right) \mathrm{d} t \mathrm{~d} \Gamma \mathrm{d} h \\
& +\int_{a}^{b} \int_{\Gamma} \int_{0}^{t^{*}-b} d(x, t+h) p\left(x, t+h ; u^{*}\right) \\
& \times\left(y(x, t ; u)-y\left(x, t ; u^{*}\right)\right) \mathrm{d} t \mathrm{~d} \Gamma \mathrm{d} h \\
& +\int_{a}^{t^{*}-t} \int_{\Gamma} \int_{t^{*}-b}^{t^{*}-a} d(x, t+h) p\left(x, t+h ; u^{*}\right) \\
& \times\left(y(x, t ; u)-y\left(x, t ; u^{*}\right)\right) \mathrm{d} t \mathrm{~d} \Gamma \mathrm{d} h \\
& -\int_{0}^{t^{*}-b} \int_{\Gamma} \frac{\partial p\left(u^{*}\right)}{\partial \eta_{A^{*}}}\left(y(x, t ; u)-y\left(x, t ; u^{*}\right)\right) \mathrm{d} \Gamma \mathrm{d} t \\
& -\int_{t^{*}-b}^{t^{*}-a} \int_{\Gamma} \frac{\partial p\left(u^{*}\right)}{\partial \eta_{A^{*}}}\left(y(x, t ; u)-y\left(x, t ; u^{*}\right)\right) \mathrm{d} \Gamma \mathrm{d} t \\
& -\int_{t^{*}-a}^{t^{*}} \int_{\Gamma} \frac{\partial p\left(u^{*}\right)}{\partial \eta_{A^{*}}}\left(y(x, t ; u)-y\left(x, t ; u^{*}\right)\right) \mathrm{d} \Gamma \mathrm{d} t
\end{aligned}
$$

$$
\begin{aligned}
& +\int_{a}^{b} \int_{\Omega} \int_{0}^{t^{*}-b} c(x, t+h) p\left(x, t+h ; u^{*}\right) \\
& \quad \times\left(y(x, t ; u)-y\left(x, t ; u^{*}\right)\right) \mathrm{d} t \mathrm{~d} x \mathrm{~d} h \\
& +\int_{a}^{t^{*}} \int_{\Omega}^{t} \int_{t^{*}-b}^{t^{*}-a} c(x, t+h) p\left(x, t+h ; u^{*}\right) \\
& \quad \times\left(y(x, t ; u)-y\left(x, t ; u^{*}\right)\right) \mathrm{d} t \mathrm{~d} x \mathrm{~d} h .
\end{aligned}
$$

Afterwards, using the fact that $y(x, t ; u)=$ $y\left(x, t ; u^{*}\right)=\Phi_{0}(x, t)$ for $x \in \Omega$ and $t \in[-b, 0)$ and $y(x, t ; u)=y\left(x, t ; u^{*}\right)=\Psi_{0}(x, t)$ for $x \in \Gamma$ and $t \in[-b, 0)$, we obtain

$$
\begin{gathered}
-\int_{\Omega}\left(z_{d}-y\left(t^{*} ; u^{*}\right)\right)\left(y\left(t^{*} ; u\right)-y\left(t^{*} ; u^{*}\right)\right) \mathrm{d} x \\
=\int_{0} \int_{\Omega} p\left(u^{*}\right)\left(u-u^{*}\right) \mathrm{d} x \mathrm{~d} t .
\end{gathered}
$$

Substituting (63) into (35) gives

$$
\int_{0}^{t^{*}} \int_{\Omega} p\left(u^{*}\right)\left(u-u^{*}\right) \mathrm{d} x \mathrm{~d} t \leq 0, \quad \forall u \in U_{Q_{\mathrm{ad}}} .
$$

The foregoing result is now summarized.

Theorem 3. The optimal control $u^{*}$ is characterized by the condition (64). Moreover, in the particular case

$$
u^{*}(x, t)=\operatorname{sign}\left(p\left(x, t ; u^{*}\right)\right), \quad x \in \Omega, t \in\left(0, t^{*}\right)
$$

whenever $p\left(u^{*}\right)$ is nonzero.

This property leads to the following result.

Theorem 4. If the functions $c(x, t)$ and $d(x, t)$ are analytic and $\Omega$ has analytic boundary $\Gamma$, then there exists a unique optimal control for the mixed initial-boundary value problem (6)-(11). Moreover, the optimal control is bang-bang, that is, $\left|u^{*}(x, t)\right| \equiv 1$, almost everywhere and it is the unique solution of (6)-(11), (47)-(54), (65).

The sketch of the proof of Theorem 4 is the same as in the case of Theorem 2.3 in (Knowles, 1978). Indeed, if we show that $p(x, t) \neq 0$ for almost all $(x, t) \in$ $\Omega \times\left(0, t^{*}\right)$, then Theorem 4 follows from Theorem 3 . This condition can be proved using properties of analyticity (Tanabe, 1965), continuity (Lions, 1971) and backward uniqueness (Friedman, 1969) for $p(u)$, respectively.

\section{Generalizations}

The time optimal control problems presented here can be extended to certain cases of nonlinear control without convexity. Such extension can be applied to solving many nonlinear control problems in mechanical engineering. 
For this purpose, suppose that a Lebesgue measurable set-valued mapping $F: \Omega \times[0, T] \rightarrow C \mathbb{R}$ (compact subset of $\mathbb{R}$ ) is given, for which

$$
\max |F(x, t)| \leq g(x, t),(x, t) \in \Omega \times[0, T]
$$

and $g \in H^{0,1}(Q)$.

Let the set of admissible controls be

$$
\begin{aligned}
\mathcal{L}_{F}=\{u: & u \text { is Lebesgue measurable, } \\
& u(x, t) \in F(x, t),(x, t) \in Q\} .
\end{aligned}
$$

The assumption (66) implies that $\mathcal{L}_{F} \subset H^{0,1}(Q)$; and so, by Theorem $1, y(u) \in H^{\infty, 2}(Q)$, for each $u \in$ $\mathcal{L}_{F}$. We shall also use the fact that the set-valued mapping

$$
\mathrm{CO} F:(x, t) \rightarrow \operatorname{CO}(F(x, t))
$$

(where 'CO' denotes the convex hull) is also Lebesgue measurable, with compact, convex values. Using the results of (Casting, 1967), the following lemma can be proved.

Lemma 3. $\quad \mathcal{L}_{\mathrm{co} F}$ is a weakly compact, convex subset of $H^{0,1}(Q)$. In place of (34), we will now assume the following:

There exists $a T>0$ and $u \in \mathcal{L}_{\mathrm{co} F}$ with

$$
\left(y(T ; u), \frac{\partial y(T ; u)}{\partial t}\right) \in W .
$$

Then, we have the following theorem.

Theorem 5. If (69) holds and the functions $c(x, t)$ and $d(x, t)$ are analytic, and $\Omega$ has analytic boundary, then $W$ is hit in minimum time $t^{*}$ by a unique admissible control $u^{*} \in \mathcal{L}_{F}$ and

$$
\int_{0}^{t^{*}} \int_{\Omega} p\left(x, t ; u^{*}\right)\left(u(x, t)-u^{*}(x, t)\right) \mathrm{d} x \mathrm{~d} t \leq 0
$$

for all $u \in \mathcal{L}_{\mathrm{co} F}$. Further, $u^{*}$ is bang-bang, in the sense that $u^{*}(x, t) \in \operatorname{ex}(F,(x, t))$ for almost all $(x, t)$, where 'ex' denotes extreme points.

Proof. Consider first the control problem of steering (6) (11) to the set $W$ in minimum time, with controls $u \in$ $\mathcal{L}_{\mathrm{CO} F}$. For this problem, we can argue exactly as before, using the weak compactness and convexity of $\mathcal{L}_{\mathrm{co} F}$ and the regularity of $y(u) \in H^{\infty, 2}(Q)$ to verify the existence of an optimal control $u^{*} \in \mathcal{L}_{\mathrm{co} F}$ hitting $W$ in minimum time $t^{*}$, for which (70) holds. If we can verify that $u^{*} \in$ $\mathcal{L}_{F}$, then it must be optimal for the original problem. We shall do this by showing that $u^{*}$ is bang-bang.

For this purpose, suppose that $u^{*} \in / \operatorname{ex}\left(\mathcal{L}_{\mathrm{co} F}\right)$. Then, there exists a nonzero function $f \in L^{2}\left(\Omega \times\left(0, t^{*}\right)\right)$ for which $u \pm f \in \mathcal{L}_{\mathrm{co} F}$. Let $f(x, t) \neq 0$ for $(x, t) \in$ $E \subset \Omega \times\left(0, t^{*}\right), E$ being nonnull. For any measurable set $H \subset E$,

$$
u^{*} \pm f \chi_{\mathrm{H}} \in \mathcal{L}_{\mathrm{co} F}
$$

and so, by (70),

$$
\int_{0}^{t^{*}} \int_{\Omega} p\left(u^{*}\right)\left(u^{*}-\left(u^{*} \pm f \cdot \chi_{\mathrm{H}}\right)\right) \mathrm{d} x \mathrm{~d} t \leq 0,
$$

that is,

$$
\int_{H} p\left(u^{*}\right) f \mathrm{~d} x \mathrm{~d} t=0 \text { for any measurable set } H \subset E .
$$

The only way this can happen is for $p\left(u^{*}\right)=0$ on $E$, but this contradicts the Theorem 4 Accordingly, $u^{*} \in \operatorname{ex}\left(\mathcal{L}_{\mathrm{co} F}\right)$; and by the results of (Olech, 1996; Theorem 7.1)

$$
u^{*}(x, t) \in \operatorname{ex}(\operatorname{co} F(x, t)) \text { for almost all }(x, t) .
$$

However,

$$
\operatorname{ex}(\operatorname{co} F(x, t)) \subset \operatorname{ex} F(x, t),
$$

(Dunford and Schwartz, 1958), and so

$$
u^{*}(x, t) \in \operatorname{ex} F(x, t) \quad \text { a.e. }
$$

The uniqueness of $u^{*}$ now follows in the usual way.

Theorem 5 constitutes one of the main results of the paper. As one application of Theorem 5, we shall present the following example.

Example 1. Consider now the following problem of nonlinear control, which is of interest in certain problems of vibrating beam.

Suppose now that 6 is replaced by

$$
\begin{array}{r}
\frac{\partial^{2} y}{\partial t^{2}}+A y+\int_{a}^{b} c(x, t) y(x, t-h) \mathrm{d} h \\
=\Phi(x, t ; u(x, t)),
\end{array}
$$

where $\Phi$ is continuous in the third variable, measurable in the first two variables, and the controls $u$ are measurable and take values in a fixed compact set $U \subset \mathbb{R}$.

Suppose also that

$$
\left|\Phi\left(x, t, f_{1}\right)\right| \leq g(x, t) \text { for all }(x, t) \in Q, \text { all } f_{1} \in U
$$

for some $g \in H^{0,1}(Q)$. Then, the set-valued function $F$ : $(x, t) \rightarrow \Phi(x, t, U)$ is measurable, has compact values, and

$$
\begin{aligned}
\mathcal{L}_{F}=\{\Phi(x, t, u(x, t)): u \text { is measurable } \\
\text { and } u(x, t) \in U \text { a.e. }\}
\end{aligned}
$$




\section{(Casting, 1967).}

Consequently, if the analyticity assumptions of Theorem 5 are satisfied, the time optimal control problem (76), (7)-(11) has a bang-bang solution.

Finally, one may consider the following example.

Example 2. The condition (34) plays a fundamental role in controllability problems for time-delay infinite order hyperbolic systems. With regard to the condition (34), we can show that (6)-(11) is approximately controllable in $L^{2}(\Omega)$ in any finite time $T>0$, i.e.,

$$
\left\{\left(y(T ; u), \frac{\partial y(T ; u)}{\partial t}\right): u \in H^{0,1}(Q)\right\}
$$

is dense in $L^{2}(\Omega)$. By the Hahn-Banach theorem, this will be the case if

$$
\int_{\Omega} z_{1}(x) y(x, T ; u) \mathrm{d} x=0, \quad z_{1} \in L^{2}(\Omega),
$$

for all $u \in H^{0,1}(Q)$, implies that $z_{1}=0$. Let $p \in$ $H^{\infty, 2}(Q)$ be the unique solution of (47)-(54) with

$$
p^{\prime}(x, T)=z_{1}(x), \quad x \in \Omega .
$$

The proof of Theorem 2 showed that

$$
\int_{\Omega} z_{1}\left(y(u)-y\left(u_{1}\right)\right) \mathrm{d} x=\int_{0}^{T} \int_{\Omega} p\left(u-u_{1}\right) \mathrm{d} x \mathrm{~d} t
$$

and so, if (78) holds for all $u \in H^{0,1}(Q)$, then

$$
\int_{0}^{T} \int_{\Omega} p u \mathrm{~d} x \mathrm{~d} t=0,
$$

$u \in H^{0,1}(Q)$, and $p=0$ in $Q$.

By continuity,

$$
p^{\prime}(x, T)=z_{1}(x)=0
$$

for almost all $x \in \Omega$.

Moreover, the hyperbolic nature of (6)-11) with $u=$ $B u$ (where $B=I$ ) suggests exact controllability of (6) (11).

\section{Conclusions}

In this paper we have investigated and solved the timeoptimal control problem for time-delay infinite order hyperbolic systems with non-homogeneous Neumann boundary conditions.

We can also consider the analogous minimum time problem for such systems with non-homogeneous Dirichlet boundary conditions.
Finally, we can consider the time-optimal boundary control problem for infinite order hyperbolic systems with time delays.

The ideas mentioned above will be developed in forthcoming papers.

\section{Acknowledgment}

The research presented here was carried out within the research programme of the AGH University of Science and Technology no. 10.10.120.31.

The author would like to express his gratitude to the anonymous reviewers for their very valuable remarks.

\section{References}

Casting, C. (1967). Sur les multi-applications measurables, Revue Francaise d'Informatique et de Recherche Operationelle 1: 91-126.

Choquet, G. (1969). Lectures on Analysis, Vol. 2, W.A. Benjamin, New York, NY.

Dubinskij, J. A. (1975). Sobolev spaces of infinite order and behavior of solution of some boundary value problems with unbounded increase of the order of the equation, Matiematiczeskii Sbornik 98: 163-184.

Dubinskij, J. A. (1976). Non-trivality of Sobolev spaces of infinite order for a full euclidean space and a torus, Matiematiczeskii Sbornik 100: 436-446.

Dubinskij, J. A. (1986). Sobolev Spaces of Infinite Order and Differential Equations, Teubner-Texte zur Mathematik, Vol. 87, Teubner-Verlag, Leipzig.

Dunford, N. and Schwartz, J. (1958). Linear Operators, Vol. 1, John Wiley and Sons, New York, NY.

El-Saify, H. A. and Bahaa, G. M. (2002). Optimal control for $n \times n$ hyperbolic systems involving operators of infinite order, Mathematica Slovaca 52: 409-422.

Friedman, A. (1969). Partial Differential Equations, Holt, Reinhart and Winston, New York, NY.

Knowles, G. (1978). Time optimal control of parabolic systems with boundary conditions involving time delays, Journal of Optimization Theory and Applications 25(4): 563-574.

Kowalewski, A. (1993a). Boundary control of hyperbolic system with time lags, IMA Journal of Mathematical Control and Information 10: 261-272.

Kowalewski, A. (1993b). Optimal control of hyperbolic system with time lags, Applied Mathematics and Computer Science 3(4): 687-697.

Kowalewski, A. (1995). Optimal control of hyperbolic system with time-varying lags, IMA Journal of Mathematical Control and Information 12: 133-143.

Kowalewski, A. (1998). Optimal control of a distributed hyperbolic system with multiple time-varying lags, International Journal of Control 71: 419-435. 
Kowalewski, A. (2000). Optimal control of distributed hyperbolic systems with deviating arguments, International Journal of Control 73: 1026-1041.

Kowalewski, A. (2003). Time-optimal control problem of hyperbolic systems with deviating arguments, International Journal of Control 76: 557-565.

Lions, J. (1971). Optimal Control of Systems Governed by Partial Differential Equations, Springer-Verlag, Berlin/Heidelberg.

Lions, J. and Magenes, E. (1972). Non-Homogeneous Boundary Value Problems and Applications, Vols. 1 and 2, SpringerVerlag, Berlin/Heidelberg.

Olech, C. (1966). Extremal solutions of a control system, Journal of Differential Equations 2: 74-101.

Tanabe, H. (1965). On differentiability and analyticity of weighted elliptic boundary-value problems, Osaka Mathematical Journal 2: 163-190.

Wang, P. K. C. (1975). Optimal control of parabolic systems with boundary conditions involving time delays, SIAM Journal of Control 13(2): 274-293.

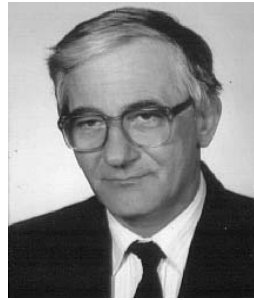

Adam Kowalewski was born in Cracow, Poland, in 1949. He received his M.Sc. degree in electrical engineering and his Ph.D. and D.Sc. degrees in control engineering from the AGH University of Science and Technology in Cracow in 1972, 1977 and 1992, respectively. At present he is a professor of automatic control and optimization theory at the Faculty of Electrical Engineering, Automatics, Computer Science and Electronics of the AGH University of Science and Technology. Professor Kowalewski is also a member of the Optimization and Control Section within the Committee of Mechanics of the Polish Academy of Sciences.

Received: 28 October 2008 